\title{
The Rationale for the Automation of a New Diagnostic Thermography Protocol to Confirm a Chronic-Low-Back-Pain Subtype Related to Nociplastic Pain
}

\author{
Elzbieta Skorupska ${ }^{1,2, *(D)}$ and Tomasz Dybek ${ }^{2}$ \\ 1 Department of Physiotherapy, Poznan University of Medical Sciences, 61-701 Poznan, Poland \\ 2 Faculty of Physical Education and Physiotherapy, Opole University of Technology, 45-271 Opole, Poland; \\ dtdybek@gmail.com \\ * Correspondence: skorupska@ump.edu.pl
}

Citation: Skorupska, E.; Dybek, T. The Rationale for the Automation of a New Diagnostic Thermography Protocol to Confirm a Chronic-LowBack-Pain Subtype Related to Nociplastic Pain. BioMed 2021, 1 , 99-111. https://doi.org/ 10.3390/biomed1020009

Academic Editor: Marco Rengo

Received: 29 May 2021

Accepted: 27 September 2021

Published: 12 October 2021

Publisher's Note: MDPI stays neutral with regard to jurisdictional claims in published maps and institutional affiliations.

Copyright: (c) 2021 by the authors. Licensee MDPI, Basel, Switzerland. This article is an open access article distributed under the terms and conditions of the Creative Commons Attribution (CC BY) license (https:// creativecommons.org/licenses/by/ $4.0 /)$.

\begin{abstract}
Gluteal syndrome (GS), a new low-back-pain subtype mimicking sciatica, has been included in the 11th Revision of the International Classification of Diseases (ICD-11). Low back pain is a symptom, not a disease, and the main problem associated with it is pain complexity. A plausible pain generator of gluteal syndrome is the central sensitization process and the therapeutic target area, which are trigger points located within the gluteal muscles. It has been hypothesized that dysregulated immune and autonomic nervous systems (ANS) are involved in central sensitization development. Changes in ANS regulation, mainly through the sympathetic branch, provoke nociceptor activation indirectly by a vasoconstriction-vasodilatation imbalance, or directly by sympatheticnociceptor activation resulting in widespread pain, hyperalgesia, and allodynia. The minimally invasive procedure (MIP) uses thermography to confirm a completely new biological phenomenon, which suggests a pathological autonomic response to noxious stimuli and can possibly become an objective marker of some nociplastic pain subtypes related to trigger points. This review provides the biological and technical rationale for the automation of the MIP-a possible future diagnostic tool for an objective gluteal syndrome confirmation.
\end{abstract}

Keywords: trigger points; gluteal syndrome; central sensitization; MATLAB; sciatica; infrared thermography camera; active dynamic thermography

\section{Introduction}

The year 2021 has been announced by the International Association for the Study of Pain (IASP) as "The Global Year About Back Pain". This initiative is aimed at developing an understanding of the nature of back pain and the utility of available treatment modalities. Low back pain (LBP) is reported by people of all ages and experienced by up to $84 \%$ of the adult population [1,2]. LBP is a symptom, not a disease, and the main problem is pain complexity. Thus, a diagnostic evaluation is challenging and requires complex clinical decisions to identify a plausible pain source before the right therapeutic approach can be determined [2]. Lately, the increasing prevalence of acute and chronic LBP, which doubled in the aging population, has been underlined [3]. This leads to activity limitation for the patients and their temporary working disability, as well as results in medical burden and economic cost [4-6]. The most problematic subgroups are patients developing the chronic form characteristic of around $10 \%$ of LBP cases [7]. It seems especially important in the light of the 11th revision of the International Statistical Classification of Diseases and Related Health Problems (ICD-11), where chronic pain has been categorized for the first time as a separate disease [8,9]. Two categories have been distinguished, namely, chronic primary musculoskeletal pain and chronic secondary musculoskeletal pain. The first pain category is recognized when the pain is a disease by itself, covering all chronic pain states in muscles, joints, or tendons that cannot be referred to any by known disease or damage 
process. Secondary musculoskeletal pain is when the pain is progressing to chronicity due to some underlying condition and assumes persistent local or systemic inflammation due to infection, crystal deposition, autoimmune or autoinflammatory processes, local structural musculoskeletal changes, or nervous system diseases not classified as musculoskeletal but causing symptoms that can provoke musculoskeletal problems (e.g., hypertonicity in Parkinson disease or spasticity in multiple sclerosis).

Another clinical aspect of LBP is pain followed by leg pain diagnosed as radicular pain, sciatica, facet joint syndrome, sacroiliac joint pain, etc. [10]. Importantly, a new subtype of LBP with leg pain, namely, gluteal syndrome (GS) mimicking sciatica, has been included in the ICD-11 [9]. This new LBP subtype is categorized as a local syndrome of the lower limbs provoked by the myofascial pain from trigger points located in one of the three gluteal muscles (i.e., gluteus maximus, medius, or minimus) and perceived as sciatica. GS can exist as a separate disease or can overlap with real sciatica. Lasegue sign can be positive, and there is no information as regards other diagnostic tools conservatively used to confirm sciatica. However, the utility of standard neurological examinations and magnetic resonance imaging in distinguishing sciatica from other pain syndromes has been questioned [11,12]. Moreover, the palpatory criteria commonly used for trigger points diagnosis are regarded as not objective and thus they are also widely questioned [13,14]. Unfortunately, this means that the new LBP subtype is likely to become another pain syndrome that is difficult to manage. This is of great concern, especially if we realize that the gluteal muscles, not considered before as the source of "sciatic pain", are the therapeutic area for GS.

One of the IASP's ideas behind the announced "Global Year About Back Pain" is the integration of tools used for stratifying people with back pain and dividing them into management pathways. An attempt to subgroup pain patients following the three main pain pathomechanisms, namely, nociceptive, neuropathic, and nociplastic, has been proposed for future studies [9]. A plausible pain generator of gluteal syndrome is the central sensitization process (nociplastic pain). There is a lack of objective tools to confirm the central sensitization (CS) process [15]. One of the new diagnostic ideas for CS patients (nociplastic pain) is to check the utility of autonomic nervous system (ANS) examination. This is relevant, especially for chronic LBP, where the ANS importance was highlighted [16,17].

The aim of this review is to provide the biological and technical rationale for the minimally invasive procedure (MIP), which could become a possible future diagnostic tool for an objective confirmation of nociplastic pain related to muscles, e.g., gluteal syndrome.

\section{Autonomic Nervous System-A Possible Marker of Nociplastic Pain Involvement}

Regardless of all the scientific data and chronic pain legitimization, the most important thing is to establish an objective nociplastic pain diagnosis. Currently, the recommended methods include the self-reported central sensitization inventory questionnaire and an array of objective tests, i.e., quantitative sensory testing (QST). The ANS involvement measurement in any pain states, e.g., to measure pain severity, was abandoned some time ago. However, some data indicate its diagnostic utility in cases when noxious stimulation is involved, and immediate ANS imbalance reactions are registered [18-20]. Heart rate variability (HRV) has been proposed to support the central sensitization examination [21,22]. It has remarkable potential, especially for nociplastic pain, where ANS dysregulation is an important factor. The HRV assumes a measurement of the variations in the interval length between consecutive heart beats [19]. There are two HRV parameters that indicate different activities: low-frequency (LF)-HRV is linked to baroreflex-dependent outflow to the heart, and high-frequency (HF)-HRV reflects the vagal cardiac control. The ratio of these two parameters describes the sympathovagal balance. The vagal nerve activity is used to control and lower pain perception. For diagnostic utility, baroreceptors seem more interesting. They control and maintain the stability of blood pressure via ANS. It has been observed that the reduction of pain co-occurred with spontaneous high blood pressure, and the same reactions have been observed during mechanical baroreceptors 
stimulation [23]. Additionally, decreased baroreceptor activity has been observed in some chronic pain states.

Recent studies that focused on the ANS response to noxious stimulation in pain patients have revealed some important facts [24]. It has been proved that the sympathetic function is more related to stimulus intensity than perceived pain intensity. A similar observation has been provided by studies of a minimally invasive procedure (MIP) applied in conditions related to central sensitization [25-27]. The MIP provoked transient amplified vasomotor phenomenon defined as autonomic referred pain (AURP), which is a newly discovered biological reaction due to the ANS imbalance caused by the noxious stimulus applied to active trigger points $[25,26]$. Importantly, the AURP develops exactly in the patients' pain zone coincident with the muscle referred pain pattern proposed by Travell and Simons in their published theory of myofascial pain [13]. The MIP protocol uses infrared thermography (IRT) camera to observe the AURP development in time, not trigger point localization.

\section{The Background for the MIP Application}

Currently, trigger points are categorized as pain syndrome due to the central sensitization (CS) process involvement necessary for its development and/or maintenance $[9,14]$. The IASP defined sensitization as "increased responsiveness of nociceptive neurons to their normal input, and/or recruitment of a response to normally subthreshold inputs" [24]. Central sensitization is an overly complex phenomenon with a wide range of induction and maintenance mechanisms characterized by secondary hyperalgesia, allodynia, and/or the presence of increased temporal summation of pain [28,29]. Recently, it has been proposed that the term central sensitization be replaced with the term nociplastic pain, defined as altered nociceptive function without any symptoms of a disease or damage of the somatosensory system due to central sensitization leading to chronic pain [29]. This proposal is supported by the fact that three main pain pathomechanisms have been defined in order to stratify patients and provide more effective treatment, namely, nociceptive, neuropathic, and nociplastic pain [29]. Generally, nociceptive pain is considered as physiological, whereas the other two are considered pathological pain, and the chronic form of nociceptive pain is considered pathological as well. Furthermore, neuropathic and nociplastic pain are characterized by the abnormal functioning of the somatosensory nervous system [30]. While, according to the established neurological diagnostic criteria, neuropathic pain is due to a demonstrable lesion or disease, nociplastic pain is associated with no such signs [29]. The medical approach for nociceptive and neuropathic pain treatment is commonly known, but nociplastic pain is currently poorly understood by most specialists.

Clinically, chronic pain patients present with amplified pain perception regarding its intensity, duration, and distribution, as well as maladaptive psychosocial factors, low vagal nerve activity, or-as mentioned above-with hyperalgesia or allodynia [31]. Currently, there is no gold standard for CS diagnosis. A self-reported questionnaire and quantitative sensory testing supported by clinical examination are recommended [32]. Moreover, it has been noticed that CS can be difficult to evaluate as its manifestations vary from pain condition to pain condition [31]. Two main types of CS mechanism among chronic pain patients have been hypothesized. Hart et al. distinguished the "top-down" and "bottomup" subtypes [32]. In the "bottom-up" CS mechanism, pain is said to be perceived due to an excess noxious peripheral input that eventually sensitizes the central nervous system to the point of perceiving pain. Over time, pain is perceived even when there is no peripheral drive [32]. The "top-down" theory suggests that changes already present within the central nervous system drive the perception of pain; the primary problem may very likely originate in supraspinal structures, and no ongoing nociceptive input is required to maintain the process [33,34]. Female predisposition is common for both CS mechanisms. Otherwise, the mechanisms differ. To illustrate the difference, patients manifesting the "top-down" subtype can have a family history of pain, high psychological co-morbidity, increased sensitivity to non-pain sensory stimuli, and a high number of chronic overlapping pain 
conditions, and the beginning of their symptoms is associated with puberty. Patients with the "bottom-up" subtype are generally less burdened [32]. The most important difference between the two subtypes of CS mechanism is that "bottom-up" is thought to be a reversible process, contrary to irreversible "top-down". Because of its reversible nature, the "bottomup" CS subtype seems particularly interesting. Muscle pain related to trigger points presence seems to be an example of this CS subtype [35]. If the hypothesis about the "bottom-up" and "top-down" CS mechanisms is true, patients with different CS subtypes will need different treatment. The similarities between trigger points and both subtypes of central sensitization are shown in Table 1, which is a modification of a similar table by Hart et al. [32] (the Trigger points column and the scientific opinion and therapy rows have been added).

Table 1. The similarities of trigger points and the "bottom-up" and "top-down" subtypes of central sensitization [32].

\begin{tabular}{|c|c|c|c|}
\hline & $\begin{array}{l}\text { "Top-Down" } \\
\text { Subtype }\end{array}$ & $\begin{array}{l}\text { "Bottom-Up" } \\
\text { Subtype }\end{array}$ & Trigger Points \\
\hline Scientific opinion & hypothesis & hypothesis & some scientific evidence exists \\
\hline Sex ratio & significant female domination & female domination & female domination \\
\hline Age & young & any age & any age \\
\hline Family history of pain & yes & no & no \\
\hline Psychological co-morbidity & high & moderate & $\begin{array}{l}\text { some correlation with depression; sleep } \\
\text { deprivation }\end{array}$ \\
\hline Therapy & a multimodal approach & not defined & $\begin{array}{c}\text { manual pressure, dry needling, and } \\
\text { TENS }\end{array}$ \\
\hline
\end{tabular}

\subsection{Central Sensitization Involvement in Muscles}

Clinically, the hypersensitivity of the somatosensory system is associated with a series of symptoms such as referred pain, widespread pain, tactile allodynia, and heightened response to non-noxious stimuli. [36]. It has been hypothesized that the neuroplasticity process leading to the CS involvement in muscles is initiated by a brief burst of C-fiber activity followed by nociceptor activity provoking the excitability of central nociceptive neurons in the cortex, brain stem, trigeminal nucleus, and spinal cord [37]. Dysregulated immune and autonomic nervous systems are involved, and the key structure maintaining that state is probably the periaqueductal gray $[38,39]$. The ANS impact is considered to occur via reflexes that affect muscle circulation and contractility, sensory-motor control, and inflammatory processes. Changes in the ANS regulation, mainly through the sympathetic branch, provoke nociceptor activation indirectly by a vasoconstriction-vasodilatation imbalance or directly by sympathetic nociceptor activation, resulting in widespread pain, hyperalgesia, and allodynia [40]. A muscle pain syndrome characterized by trigger points is an example of this mechanism [34].

Two types of myalgia are linked to the central sensitization process, i.e., fibromyalgia and myofascial pain syndrome (MPS). While the former is a rheumatological disease on the ICD list, the latter is a pain syndrome recognized as functional or non-specific pain (some of the subtypes, e.g., gluteal syndrome, are included in the ICD-11 [9]). Owing to the occurrence of decreased pressure pain thresholds and referred pain, MPS is thought to be related to central sensitization, which generates an expansion of receptive fields [34]. Additionally, it has been confirmed that the referred pain area is growing exponentially with an increased degree of central sensitization [41].

Generally, myofascial pain syndrome is defined as a non-inflammatory local pain syndrome characterized by: (i) trigger points (TrPs), i.e., limited sites of severe muscle tenderness or hypersensitivity; (ii) a determined area of referred pain; and (iii) characteristic motor, sensory, and autonomic dysfunctions and symptoms [42,43]. A trigger point is an area with multiple "TrPs loci" composed of (i) a sensory component: sensitized nociceptor, i.e., free nerve ending, and (ii) a motor component: dysfunctional end-plate in the vicinity of the sensitized nociceptor [44]. From the clinical point of view, trigger points are divided 
into active, which cause myofascial pain syndrome symptoms, and latent, which refer no pain until mechanical stimulation is applied.

The autonomic nervous system involvement is also taken into consideration as regards the TrPs development. The most accepted theoretical concept of TrPs activation is Simons' integrated hypothesis, which assumes five or six steps of the positive-feedback cycle [45]. The hypothesis suggests that abnormal depolarization of the post-junctional membrane of motor end-plates causes sarcomere shortening, lower concentration of adenosine triphosphate, and localized hypoxia leading to bradykinin release and severe sensitization of intramuscular nociceptors. A higher concentration of $\mathrm{H}+$ protons, serotonin, prostaglandins, $\mathrm{K}+$ ions, and calcitonin-gene-related peptide said to sustain the irritability and dysregulation of myoneural junctions has been confirmed. There are five stages of this hypothesis: (1) abnormal spontaneous ACh release; (2) formation of the taut band; (3) local hypoxia; (4) tissue distress; and (5) sensitizing substances have been confirmed experimentally except for the last one (autonomic modulation), which assumes that the ANS affects the intensity of symptoms [45]. If the MIP findings can be confirmed for both active and latent form of trigger points, these steps can be confirmed as well.

Currently, there are only a few experimental studies suggesting the ANS involvement in muscle referred pain $[26,27]$. Those studies have documented intensive vasodilatation in the area of perceived sciatic pain provoked by a minimally invasive method applied to trigger points within gluteus minimus. The observed impaired blood flow in the pain region is said to be related to sympathetic hyperactivity and diminished parasympathetic tone $[46,47]$. Thus, the ANS dysregulation characteristic of the central sensitization process can be presumed for trigger points. However, it is not known if TrPs in other muscles would provoke a similar reaction, which-if positive-would be consistent with CS studies. The knowledge of referred pain seems crucial because its location is incompatible with both innervation and commonly known dermatomes [48-51]. Recently, the international panel of experts has promoted the revision of TrPs clinical criteria and indicated the crucial meaning of referred pain for a more valuable TrPs confirmation [52]. The referred pain pattern has a key diagnostic meaning, and it is one of the additional criteria confirming TrPs because there is a strong correlation between a given trigger point location and the referred pain pattern defined for this trigger point. Importantly, trigger point for referred pain occurs in the same area in all patients. Moreover-in the majority of cases-referred pain does not coincide with the area of the trigger point presence. That is why therapy is usually applied to an area different than the area corresponding to the daily complaint. Moreover, $\operatorname{TrPs}$ symptoms are reversible, which is compatible with Hart and coworkers' [32] hypothesis concerning the "bottom-up" CS subtype existence.

\subsection{Central Sensitization Involvement in Other Tissues Leading to Low Back Pain}

Low back pain is usually nonspecific or mechanical, arising intrinsically from the spine, intervertebral disks, or surrounding soft tissues [53]. Little is known about CS in chronic pain states related to joints, but some animal studies support its involvement [54]. Generally, CS involvement in joint pain has been indicated for osteoarthritis (OA); rheumatoid arthritis; Ehlers-Danlos syndrome; or sacroiliac joint syndrome, which mimics sciatic pain as well [55-58]. OA patients demonstrated diffuse and spreading pain to areas larger than the joint localization [59]. A specific group with mild to moderate joint damage but high pain were a particularly sensitized group of patients [60]. A specific sensitivity index may help to optimize pain management and profile patients prior to joint replacement surgery. Sensitization may be one of the factors involved in the development of chronic postoperative pain after joint replacement [61].

Moreover, joints can develop referred pain and present secondary hyperalgesia, considered an important CS feature. The sacroiliac joint syndrome provokes a pain pattern originating from ligaments similar to lumbosacral disorders [62]. Unfortunately, the pain mechanism for that syndrome is still unknown [63]. The CS involvement is considered for inflammatory joint pain as well. It has been suggested that the CS mechanism can provide 
a neurophysiological explanation of the persistent pain in rheumatoid arthritis remission regardless of the joint degeneration process [55].

Currently, there is no hypothesis regarding the mechanism that could possibly provoke the CS development in joints. A process similar to the one occurring in muscles might be assumed [64]. Some data have indicated that ANS can influence joints as well. Both sympathetic and sensory nerves have been confirmed within vascular channels of the osteoarthritis cartilage, which is not observed in healthy joints [65].

\section{The Rationale for the Automation of the Minimally Invasive Procedure for Confirming Gluteal Syndrome Objectively}

One of the technologies applied in modern medicine is imaging performed with an infrared thermography (IRT) camera. Thermography has been used for years to assess different diseases, e.g., breast cancer, severe acute respiratory syndrome, diabetic disorders, Raynaud phenomenon, and neuropathic pain. [66]. The biological background for IRT measurement is based on complex reactions between blood-flow rate and local structures of subcutaneous tissues with the primary role of the sympathetic nervous system activity. Skin temperatures in healthy individuals are symmetrical [67]. Thus, thermal symmetry assessment is considered a valuable method to assess physiological normality/abnormality. Additionally, diseases can provoke local or systemic effects on thermoregulation, resulting in changes detectable by IRT [68]. Thus, thermography is applied in order to: (i) identify temperature asymmetry; (ii) provide a direct assessment to confirm dysfunctional autonomic, sympathetic, and parasympathetic physiological activations due to the presence of a disease; (iii) compute physiological information: localized blood flow, cardiac pulse, etc., to non-invasively detect the location of a geometrical shape or vascular/focal pattern, e.g., tumor; and (iv) provide the data for the underlying alterations in the main thermoregulatory functions showing environmental heat exchange process, vasoconstriction and/or vasodilatation, and sweating actions [69].

For biomedical applications, two methods of thermography measurement are used: passive and active dynamic thermography $[70,71]$. Out of these two methods, active dynamic thermography (ADT), where an external excitation or stimulation by cold, hot, or pressure is applied to the target area to provoke more intensive, transient biological reactions resulting in high contrast thermal images, provides more valuable diagnostic data. Most of the ADT methods are aimed at obtaining a high-quality single thermal picture for further quantitative analysis performed with specific software [72].

The MIP protocol is a proposal of a completely new approach in medical thermography. It is similar to ADT protocols, but it has some basic differences [25,26,29]. Generally, the MIP allows one to register pathological ANS activity, which can help characterize a certain subpopulation of pain patients. This autonomic phenomenon is a new biological finding specific to trigger points exclusively. More precisely, the innovative solutions applied in the MIP are as follows: (i) the method is focused on a specific subtype of pain (nociplastic pain related to muscle), not any pain; (ii) an invasive noxious stimulant (nociceptive muscle needling - fast-in fast-out dry needing technique widely used as a therapeutic tool) is applied for the first time; (iii) the analysis is performed on one side of the body only (just the painful side, without considering the opposite side); (iv) the analysis is based on all 320 thermograms, not one; (v) the pathological region is indicated by software, not a technician; and (vi) the diagnosis is confirmed if a simultaneous significant increase (vasodilatation)/decrease (vasoconstriction) in both MIP diagnostic parameters is observed in a series of pictures.

The method has been validated, and the published data confirmed amplified vasodilatation is limited to the referred pain zone of the stimulated gluteus minimus trigger points diagnosed for sciatica or low-back-leg-pain subjects [25]. According to the validation study, the two parameters, namely, (i) the significant increase in the average temperature and (ii) the AURP development, were indicated as diagnostically valuable. The second parameter allows the visualization of the patient's pain area (transient, amplified vasodilatation) as shown in Figure 1b. The trigger points studies using the MIP on sciatica and low-back-leg- 
pain patients showed the AURP size that ranged from 5\% to approximately $30 \%$ of the lower limb with an average temperature increase of above $1.5^{\circ} \mathrm{C}[26,27]$. The newly published data indicated the leading diagnostic value of the significant average temperature $(\Delta$ Tavr) increase within the perceived pain area covered by the AURP. To confirm a gluteus minimus trigger point objectively, a simultaneous significant increase in both diagnostic parameters needs to be proved [29].

The manual thermal data analysis was the main disadvantage of the MIP. The procedure allows one to record thermograms every $3 \mathrm{~s}$, but the published data presented only three representatives thermograms of the MIP stages, i.e., one of the initial states, one of the states after $10 \mathrm{~min}$ of the nociceptive muscle stimulation, and finally one of the states $6 \mathrm{~min}$ post-stimulation seen during the observation phase $[25,26,29]$. Thus, a lot of valuable data were overlooked, e.g., the AURP development visible in the video file [28] suggested some fluctuations, which would demand the analysis of the whole (around 320) thermogram series recorded during the MIP. Additionally, a detailed description of the recovery phase defining the quenching time of the reactions provoked by stimulation is one of the fundamental terms of each ADT protocol [72]. It would be very time-consuming to manually analyze all of the 320 thermograms recorded for each patient during the MIP. Furthermore, it would be impossible to perform such a manual analysis for a big group of patients. All these aspects were considered in the study presenting the MIP automation [29].

The MATLAB (version R2021) analysis showed the necessity of correcting the length of the MIP phases [29]. It seems possible to shorten the noxious stimulation phase, but further studies considering this aspect are necessary. The observation phase seems too short. The current protocol allowed us to confirm the moment of the maximum autonomic phenomenon development but was not enough to observe the patient's return to the norm from before the stimulation. According to active dynamic thermography protocols, the MIP method should have a precise definition of the recovery phase [30]. Two ways are possible to improve the protocol as regards the recovery phase. One is the lengthening of the observation phase, and the other is the shortening of the stimulation phase. Both ways can probably lead to a faster recovery. The latter seems more viable because it would shorten the time of the painful stimulation phase. Further studies considering the time demanded for both the stimulation and observation phase are necessary.

The automated quantitative analysis makes thermography more objective and reliable and allows the processing of big data [73]. The use of the MATLAB software for the analysis of thermograms recorded during the MIP renders the procedure results faster and provides lots of additional information concerning the AURP and temperature changes that depend on the time of the procedure and the clinical state of the patient. For further MIP development supported by MATLAB, a precise determination of the region of interest (ROI) can be reconsidered. Presently, the MIP considered anatomical ROIs automatically defined by MATLAB. Within those ROIs, the software segmented the picture towards the AURP size according to the MATLAB codes specifically established for the MIP [29]. 
(I) Gluteus minimus muscle referred pain pattern established by Travell \& Simons

(drawn based on the patient's report)

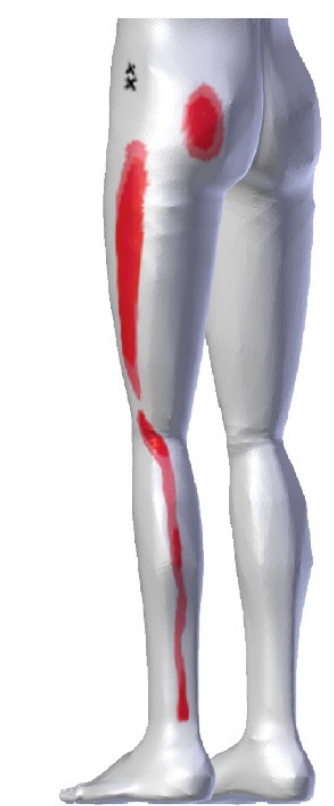

(II) Diagnostic protocol

\section{Minimally Invasive Procedure (MIP)}

A. $10^{\prime}$ muscle noxious stimulation under infrared thermography (IRT) camera control (stimulation phase)

B. $6^{\prime}$ further IRT observation of the patient at rest (observation phase)

C. MATLAB analysis of the recorded 320 thermograms

Gluteal syndrome was confirmed if the patients presented pathological autonomic reactivity within the perceived pain zone manifested by a simultaneous significant increase in both MIP diagnostic parameters.

MIP diagnostic parameters (AURP and $\triangle T$ Tavr)

1. both significantly increased - vasodilatation 2. both significantly decreased - vasoconstriction

(III) MIP-based objective confirmation of nociplastic pain related to the gluteus minimus muscle

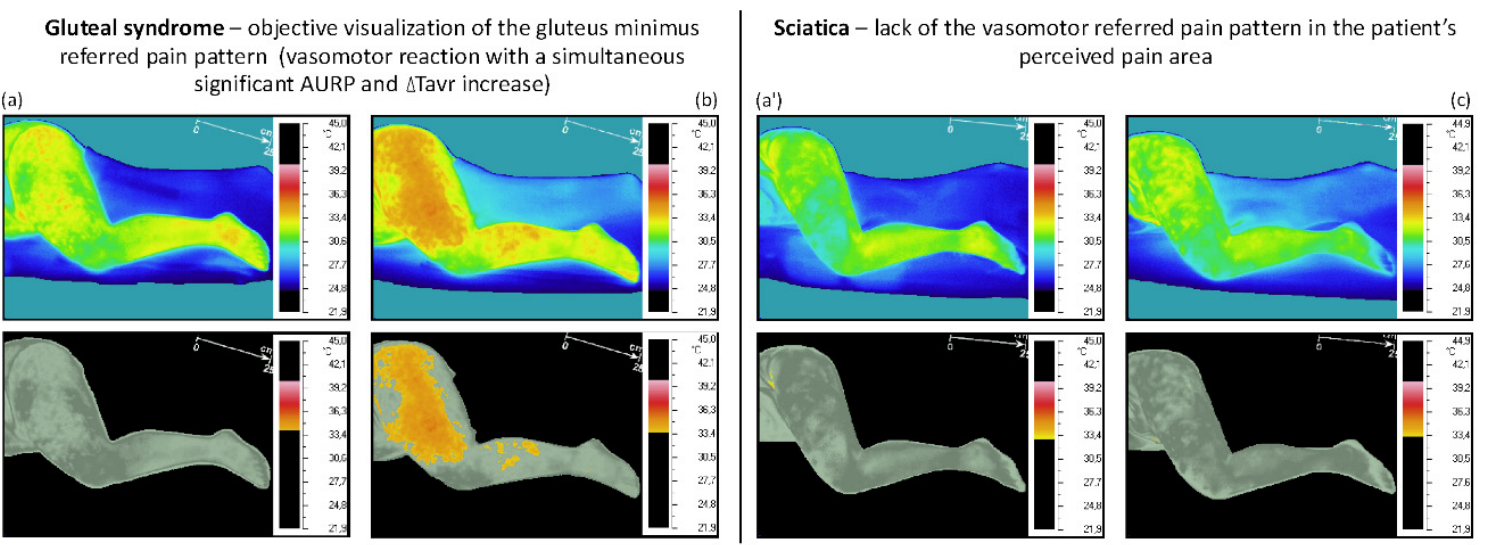

Figure 1. An illustration of the minimally invasive procedure (MIP) applied to a gluteal syndrome case (vasodilatation) and a chronic sciatica case (lack of a significant vasomotor reactivity). (I) gluteus minimus referred pain pattern established by Travell and Simons based on the post-stimulation reports of the patients with gluteus minimus trigger points (not objectively confirmed); (II) diagnostic protocol; and (III) examples of the registered 320 MIP thermograms (a) and (a') pictures of a patient before noxious stimulation (patients at rest); basal thermogram, (b) pictures showing positive MIP results-a registered pathological ANS activity within the patient's perceived pain zone, and (c) pictures showing negative MIP results-lack of a pathological ANS activity within the patient's perceived pain zone (color picture-full range of temperature; gray picture-individually isolated state above the maximum temperature at rest).

It seems that this proposed ROIs determination is optimal because currently established solutions for medical thermography cannot be directly applied to the MIP [74]. The Glamorgan Protocol published in 2008 standardized 90 different ROIs in thermographic studies [72]. Nevertheless, even though the Glamorgan Protocol provides proper guidance as to the ROI determination, it is not helpful as regards the MIP. Many IRT studies have developed their criteria for creating and selecting ROIs. There are some studies with very specific demands owing to different ROI geometries and methodologies [75-77]. Thus, it would be necessary to specify our own ROI for the MIP in the future, e.g., specific for 
each of the trigger points patterns. However, it is difficult to determine the ROI due to different body types of individuals (such us Ectomorphs, Endomorphs, Mesomorphs, or a combination of them) and personal anthropometric dimensions (e.g., different leg length, size), which results in different sizes and shapes of the observed area. An additional difficulty is the positioning of the patient on the couch and a slightly different position in relation to the camera. Besides, a slight movement due to pain occurrence as a result of noxious stimulation of the affected nociceptors ( $\mathrm{TrPs}$ ) is yet another problem to solve. In general, this is one of the most controversial points regarding IRT application in humans [68]. One solution is to define anatomical ROI and then use image segmentation with a defined temperature range to interpret the AURP development reflecting the referred pain zone of the examined trigger point.

Another aspect is the reproducibility of the ROI determination for different images to ensure the accuracy of the results, especially when different subjects are compared [68]. Various strategies are used to increase the reproducibility of thermographic data, e.g., the software with an automatic ROI selection [78-80]. For the MIP, two types of automated thermogram analysis were assumed. According to Ludwig et al. [81], the first one uses the maximum temperature to determine the ROIs. The operator selects an ROI, and the software automatically selects the five warmest pixels within the ROI, with a minimum distance of at least five pixels between each. The software chooses an area of five $x$ five pixels around each of the five pixels previously selected, determining the mean temperature across the 125 pixels included in the selection. The authors suggested that the main advantage of this method is the use of the same number of pixels for the calculation, which removes the effect of different anatomical sizes between participants. The second way to determine the ROIs is using the markers in anatomical references or outer delimiting areas of the body surface (called a mask) [79]. This strategy is useful in ensuring the reproducibility of the ROI delimitation, and it can facilitate further data analysis. The new MIP protocol supported by MATLAB, version R2021, used both solutions for the calculation of the two MIP parameters and a further comparison between the subjects.

\section{Limitations}

A disadvantage of the MIP is painful stimulation, which is necessary to provoke pathological ANS reactivity within trigger points referred pain. The minimum time of noxious stimulation and the time of the observation phase need to be defined to build a precise protocol according to the active dynamic thermography requirements.

\section{Conclusions}

The minimally invasive procedure is intended to confirm a completely new biological phenomenon, which suggests a pathological autonomic response to noxious stimuli and can possibly become an objective marker of some nociplastic pain subtypes. It applies a new type of the active dynamic thermography method and uses invasive nociceptive muscle stimulation for the first time. The MIP protocol is based on the analysis of one side only and focuses on the confirmation or not of a pathological autonomic phenomenon occurrence within the perceived pain zone. Furthermore, automated thermogram picture segmentation is performed to confirm the pathology. Finally, the diagnosis is based on the analysis of all 320 thermograms using MATLAB. The first pain syndrome examined by the MIP is a new subtype of low back pain, namely, gluteal syndrome. Further studies considering the examination of other pain syndromes related to trigger points using the MIP supported by MATLAB are recommended.

Author Contributions: E.S. and T.D. contributed to data analysis, drafting, and revision of the article; gave final approval of the version to be published; and agreed to be accountable for all aspects of the work. All authors have read and agreed to the published version of the manuscript.

Funding: This research received no external funding.

Institutional Review Board Statement: Not applicable. 


\section{Informed Consent Statement: Not applicable.}

Data Availability Statement: The data presented in this study are available on request from the corresponding author. The data are not publicly available due to medical data privacy of the patients examined by MIP.

Conflicts of Interest: The authors declare no conflict of interest.

$\begin{array}{ll}\text { Abbreviations } \\ \text { LBP } & \text { low back pain } \\ \text { GS } & \text { gluteal syndrome } \\ \text { IASP } & \text { the International Association for the Study of Pain } \\ \text { MIP } & \text { minimally invasive procedure } \\ \text { ANS } & \text { autonomic nervous system } \\ \text { CS } & \text { central sensitization } \\ \text { TrPs } & \text { trigger points } \\ \text { IRT } & \text { infrared thermography } \\ \text { ADT } & \text { active dynamic thermography } \\ \text { AURP } & \text { autonomic referred pain } \\ \text { ROI } & \text { region of interest }\end{array}$

\section{References}

1. Wu, A.; March, L.; Zheng, X.; Huang, J.; Wang, X.; Zhao, J.; Blyth, F.M.; Smith, E.; Buchbinder, R.; Hoy, D. Global low back pain prevalence and years lived with disability from 1990 to 2017: Estimates from the Global Burden of Disease Study 2017. Ann. Transl. Med. 2020, 8, 299. [CrossRef]

2. Maher, C.; Underwood, M.; Buchbinder, R. Non-specific low back pain. Lancet 2012, 379, 482-491. [CrossRef]

3. Maniadakis, N.; Gray, A. The economic burden of back pain in the UK. Pain 2000, 84, 95-103. [CrossRef]

4. Lee, H.; Hübscher, M.; Moseley, G.L.; Kamper, S.J.; Traeger, A.C.; Mansell, G.; McAuley, J.H. How does pain lead to disability? A systematic review and meta-analysis of mediation studies in people with back and neck pain. Pain 2015, 156, 988-997. [CrossRef]

5. Al Mazrou, S.H.; Elliott, R.A.; Knaggs, R.D.; Al Aujan, S.S. Cost-effectiveness of pain management services for chronic low back pain: A systematic review of published studies. BMC Health Serv. Res. 2020, 20, 194.

6. Carregaro, R.L.; Tottoli, C.R.; Rodrigues, D.d.S.; Bosmans, J.E.; da Silva, E.N.; van Tulder, M. Low back pain should be considered a health and research priority in Brazil: Lost productivity and healthcare costs between 2012 to 2016. PLoS ONE 2020, 15, e0230902. [CrossRef] [PubMed]

7. Meucci, R.D.; Fassa, A.G.; Faria, N.M. Prevalence of chronic low back pain: Systematic review. Rev. Saude Publica 2015, 49, 73. [CrossRef] [PubMed]

8. Treede, R.D.; Rief, W.; Barke, A.; Aziz, Q.; Bennett, M.I.; Benoliel, R.; Cohen, M.; Evers, S.; Finnerup, N.B.; First, M.B.; et al. Chronic pain as a symptom or a disease: The IASP Classification of Chronic Pain for the International Classification of Diseases (ICD-11). Pain 2019, 160, 19-27. [CrossRef] [PubMed]

9. Classification of Chronic Pain, Second Edition (Revised). Available online: https://www.iasp-pain.org/PublicationsNews/ Content.aspx?ItemNumber=1673\&navItemNumber=677 (accessed on 15 August 2021).

10. Allegri, M.; Montella, S.; Salici, F.; Valente, A.; Marchesini, M.; Compagnone, C.; Baciarello, M.; Manferdini, M.E.; Fanelli, G. Mechanisms of low back pain: A guide for diagnosis and therapy. F1000Research 2016, 5, 1530. [CrossRef]

11. Tawa, N.; Rhoda, A.; Diener, I. Accuracy of clinical neurological examination in diagnosing lumbo-sacral radiculopathy: A systematic literature review. BMC Musculoskelet. Disord. 2017, 18, 93. [CrossRef]

12. Al Nezari, N.H.; Schneiders, A.G.; Hendrick, P.A. Neurological examination of the peripheral nervous system to diagnose lumbar spinal disc herniation with suspected radiculopathy: A systematic review and meta-analysis. Spine J. 2013, 13, 657-674. [CrossRef] [PubMed]

13. Travell, J.G.; Simons, D.G. Myofascial Pain and Dysfunction: The Trigger Point Manual, 2nd ed.; Williams \& Wilkins: Baltimore, MD, USA, 1999.

14. Li, L.; Stoop, R.; Clijsen, R.; Hohenauer, E.; Fernández-de-Las-Peñas, C.; Huang, Q.; Barbero, M. Criteria Used for the Diagnosis of Myofascial Trigger Points in Clinical Trials on Physical Therapy: Updated Systematic Review. Clin. J. Pain 2020, 36, 955-967. [CrossRef] [PubMed]

15. van Griensven, H.; Schmid, A.; Trendafilova, T.; Low, M. Central Sensitization in Musculoskeletal Pain: Lost in Translation? J. Orthop. Sports Phys. Ther. 2020, 50, 592-596. [CrossRef] [PubMed]

16. El-Badawy, M.A.; El Mikkawy, D.M. Sympathetic Dysfunction in Patients with Chronic Low Back Pain and Failed Back Surgery Syndrome. Clin. J. Pain 2016, 32, 226-231. [CrossRef] 
17. Prim, J.H.; Ahn, S.; Davila, M.I.; Alexander, M.L.; McCulloch, K.L.; Fröhlich, F. Targeting the Autonomic Nervous System Balance in Patients with Chronic Low Back Pain Using Transcranial Alternating Current Stimulation: A Randomized, Crossover, Double-Blind, Placebo-Controlled Pilot Study. J. Pain Res. 2019, 12, 3265-3277. [CrossRef]

18. Bruehl, S.; Chung, O.Y. Interactions between the cardiovascular and pain regulatory systems: An updated review of mechanisms and possible alterations in chronic pain. Neurosci. Biobehav. Rev. 2004, 28, 395-414. [CrossRef] [PubMed]

19. Koenig, J.; Jarczok, M.N.; Ellis, R.J.; Hillecke, T.K.; Thayer, J.F. Heart rate variability and experimentally induced pain in healthy adults: A systematic review. Eur. J. Pain 2014, 18, 301-314. [CrossRef]

20. Terkelsen, A.J.; Andersen, O.K.; Mølgaard, H.; Hansen, J.; Jensen, T.S. Mental stress inhibits pain perception and heart rate variability but not a nociceptive withdrawal reflex. Acta Physiol. Scand. 2004, 180, 405-414. [CrossRef]

21. Ansuategui Echeita, J.; Schiphorst Preuper, H.R.; Dekker, R.; Stuive, I.; Timmerman, H.; Wolff, A.P.; Reneman, M.F. Central Sensitisation and functioning in patients with chronic low back pain: Protocol for a cross-sectional and cohort study. BMJ Open. 2020, 10, e031592. [CrossRef]

22. Hohenschurz-Schmidt, D.J.; Calcagnini, G.; Dipasquale, O.; Jackson, J.B.; Medina, S.; O’Daly, O.; O’Muircheartaigh, J.; de Lara Rubio, A.; Williams, S.C.R.; McMahon, S.B.; et al. Linking Pain Sensation to the Autonomic Nervous System: The Role of the Anterior Cingulate and Periaqueductal Gray Resting-State Networks. Front. Neurosci. 2020, 14, 147. [CrossRef]

23. Reyes del Paso, G.A.; Montoro, C.; Muñóz Ladrón de Guevara, C.; Duschek, S.; Jennings, J.R. The effect of baroreceptor stimulation on pain perception depends on the elicitation of the reflex cardiovascular response: Evidence of the interplay between the two branches of the baroreceptor system. Biol. Psychol. 2014, 101, 82-90. [CrossRef] [PubMed]

24. Nickel, M.M.; May, E.S.; Tiemann, L.; Postorino, M.; Ta Dinh, S.; Ploner, M. Autonomic responses to tonic pain are more closely related to stimulus intensity than to pain intensity. Pain 2017, 158, 2129-2136. [CrossRef] [PubMed]

25. Skorupska, E.; Rychlik, M.; Samborski, W. Validation and Test-Retest Reliability of New Thermographic Technique Called Thermovision Technique of Dry Needling for Gluteus Minimus Trigger Points in Sciatica Subjects and TrPs-Negative Healthy Volunteers. BioMed Res. Int. 2015, 2015, 546497. [CrossRef] [PubMed]

26. Skorupska, E.; Jokiel, M.; Rychlik, M.; Łochowski, R.; Kotwicka, M. Female Overrepresentation in Low Back-Related Leg Pain: A Retrospective Study of the Autonomic Response to a Minimally Invasive Procedure. J. Pain Res. 2020, 13, 3427-3435. [CrossRef]

27. Skorupska, E.; Rychlik, M.; Samborski, W. Intensive vasodilatation in the sciatic pain area after dry needling. BMC Complement. Altern. Med. 2015, 15, 72. [CrossRef]

28. Available online: https:/ / www.iasp-pain.org/terminology?navItemNumber=576\#Sensitization (accessed on 19 May 2021).

29. Trouvin, A.P.; Perrot, S. New concepts of pain. Best Pract. Res. Clin. Rheumatol. 2019, 33, 101415. [CrossRef]

30. Rekatsina, M.; Paladini, A.; Piroli, A.; Zis, P.; Pergolizzi, J.V.; Varrassi, G. Pathophysiologic Approach to Pain Therapy for Complex Pain Entities: A Narrative Review. Pain Ther. 2020, 9, 7-21. [CrossRef]

31. Arendt-Nielsen, L.; Morlion, B.; Perrot, S.; Dahan, A.; Dickenson, A.; Kress, H.G.; Wells, C.; Bouhassira, D.; Mohr Drewes, A. Assessment and manifestation of central sensitisation across different chronic pain conditions. Eur. J. Pain 2018, 22, $216-241$. [CrossRef]

32. Harte, S.E.; Harris, R.E.; Clauw, D.J. The neurobiology of central sensitization. J. Appl. Biobehav. Res. 2018, 23, e12137. [CrossRef]

33. Price, T.J.; Gold, M.S. From Mechanism to Cure: Renewing the Goal to Eliminate the Disease of Pain. Pain Med. 2018, 19, 1525-1549. [CrossRef]

34. Harper, D.E.; Schrepf, A.; Clauw, D.J. Pain Mechanisms and Centralized Pain in Temporomandibular Disorders. J. Dent. Res. 2016, 95, 1102-1108. [CrossRef]

35. Nystrom, N.A.; Freeman, M.D. Central Sensitization Is Modulated Following Trigger Point Anesthetization in Patients with Chronic Pain from Whiplash Trauma. A Double-Blind, Placebo-Controlled, Crossover Study. Pain Med. 2018, 19, 124-129. [CrossRef] [PubMed]

36. Woolf, C.J. Central sensitization: Implications for the diagnosis and treatment of pain. Pain 2011, 152 (Suppl. 3), S2-S15. [CrossRef] [PubMed]

37. Latremoliere, A.; Woolf, C.J. Central sensitization: A generator of pain hypersensitivity by central neural plasticity. J. Pain. 2009, 10, 895-926. [CrossRef] [PubMed]

38. Truini, A.; Tinelli, E.; Gerardi, M.C.; Calistri, V.; Iannuccelli, C.; La Cesa, S.; Tarsitani, L.; Mainero, C.; Sarzi-Puttini, P.; Cruccu, G.; et al. Abnormal resting state functional connectivity of the periaqueductal grey in patients with fibromyalgia. Clin. Exp. Rheumatol. 2016, 34 (Suppl. 96), S129-S133. [PubMed]

39. Yu, R.; Gollub, R.L.; Spaeth, R.; Napadow, V.; Wasan, A.; Kong, J. Disrupted functional connectivity of the periaqueductal gray in chronic low back pain. Neuroimage Clin. 2014, 6, 100-108. [CrossRef]

40. Charkoudian, N. Mechanisms and modifiers of reflex induced cutaneous vasodilation and vasoconstriction in humans. J. Appl. Physiol. 2010, 109, 1221-1228. [CrossRef] [PubMed]

41. Fernández-de-las-Peñas, C.; Schoenen, J. Chronic tension-type headache: What is new? Curr. Opin. Neurol. 2009, 22, 254-261. [CrossRef] [PubMed]

42. Phan, V.; Shah, J.; Tandon, H.; Srbely, J.; DeStefano, S.; Kumbhare, D.; Sikdar, S.; Clouse, A.; Gandhi, A.; Gerber, L. Myofascial Pain Syndrome: A Narrative Review Identifying Inconsistencies in Nomenclature. PMER 2020, 12, 916-925. [CrossRef]

43. Travell, J.G.; Simons, D. Travell, Simons E Simons' Myofascial Pain and Dysfunction; Lippincott Williams and Wilkins: Philadelphia, PA, USA, 2018. 
44. Skorupska, E.; Zawadziński, J.; Bednarek, A.; Samborski, W. Skin Resistivity Value of Upper Trapezius Latent Trigger Points. BioMed Res. Int. 2015, 2015, 351726. [CrossRef] [PubMed]

45. Simons, D.G. Review of enigmatic MTrPs as a common cause of enigmatic musculoskeletal pain and dysfunction. J. Electromyogr. Kinesiol. 2004, 14, 95-107. [CrossRef]

46. Kimura, Y.; Ge, H.-Y.; Zhang, M.; Kimura, H.; Sumikura, H.; Arendt-Nielsen, L. Evaluation of sympathetic vasoconstrictor response following nociceptive stimulation of latent myofascial trigger points in humans. Acta Physiol. 2009, 196, 411-417. [CrossRef]

47. Zhang, Y.; Ge, H.-Y.; Yue, S.-W.; Kimura, Y.; Arendt-Nielsen, L. Attenuated skin blood flow response to nociceptive stimulation of latent myofascial trigger point. Arch. Phys. Med. Rehabil. 2009, 90, 325-332. [CrossRef]

48. Mense, S. Muscle pain: Mechanisms and clinical significance. Dtsch. Ärzteblatt Int. 2008, 105, 214-219. [CrossRef]

49. Arendt-Nielsen, L.; Svensson, P. Referred Muscle Pain: Basic and Clinical Findings. Clin. J. Pain 2001, 17, 11-19. [CrossRef]

50. Graven-Nielsen, T.; Arendt-Nielsen, L. Induction and assessment of muscle pain, referred pain, and muscular hyperalgesia. Curr. Pain Headache Rep. 2003, 7, 443-451. [CrossRef] [PubMed]

51. Mense, S.; Gerwin, R.D. Introduction. In Muscle Pain: Understanding the Mechanisms; Springer: Berlin/Heidelberg, Germany, 2010.

52. Fernández-de-Las-Peñas, C.; Dommerholt, J. International Consensus on Diagnostic Criteria and Clinical Considerations of Myofascial Trigger Points: A Delphi Study. Pain Med. 2018, 19, 142-150. [CrossRef] [PubMed]

53. Will, J.S.; Bury, D.C.; Miller, J.A. Mechanical Low Back Pain. Am. Fam. Physician 2018, 98, 421-428.

54. Havelin, J.; King, T. Mechanisms Underlying Bone and Joint Pain. Curr. Osteoporos. Rep. 2018, 16, 763-771. [CrossRef]

55. Eitner, A.; Hofmann, G.O.; Schaible, H.G. Mechanisms of Osteoarthritic Pain. Studies in Humans and Experimental Models. Front. Mol. Neurosci. 2017, 10, 349. [CrossRef] [PubMed]

56. Zhang, A.; Lee, Y.C. Mechanisms for Joint Pain in Rheumatoid Arthritis (RA): From Cytokines to Central Sensitization. Curr. Osteoporos. Rep. 2018, 16, 603-610. [CrossRef]

57. Di Stefano, G.; Celletti, C.; Baron, R.; Castori, M.; Di Franco, M.; La Cesa, S.; Leone, C.; Pepe, A.; Cruccu, G.; Truini, A.; et al. Central sensitization as the mechanism underlying pain in joint hypermobility syndrome/Ehlers-Danlos syndrome, hypermobility type. Eur. J. Pain 2016, 20, 1319-1325. [CrossRef] [PubMed]

58. O'Neill, T.W.; Felson, D.T. Mechanisms of Osteoarthritis (OA) Pain. Curr. Osteoporos. Rep. 2018, 16, 611-616. [CrossRef]

59. Arendt-Nielsen, L.; Fernández-de-Las-Peñas, C.; Graven-Nielsen, T. Basic aspects of musculoskeletal pain: From acute to chronic pain. J. Man. Manip. Ther. 2011, 19, 186-193. [CrossRef]

60. Soni, A.; Wanigasekera, V.; Mezue, M.; Cooper, C.; Javaid, M.K.; Price, A.J.; Tracey, I. Both profiling and subgrouping patients with knee osteoarthritis pain seem important. A specific group with mild to moderate joint damage, but severe pain, might be a particularly sensitized group of patients. Arthritis Rheumatol. 2019, 71, 550-560. [CrossRef] [PubMed]

61. Baert, I.A.; Lluch, E.; Mulder, T.; Nijs, J.; Noten, S.; Meeus, M. Does pre-surgical central modulation of pain influence outcome after total knee replacement? A systematic review. Osteoarthr. Cartil. 2016, 24, 213-223. [CrossRef] [PubMed]

62. Falowski, S.; Sayed, D.; Pope, J.; Patterson, D.; Fishman, M.; Gupta, M.; Mehta, P. A Review and Algorithm in the Diagnosis and Treatment of Sacroiliac Joint Pain. J. Pain Res. 2020, 13, 3337-3348. [CrossRef]

63. Eloqayli, H. Clinical Decision-Making in Chronic Spine Pain: Dilemma of Image-Based Diagnosis of Degenerative Spine and Generation Mechanisms for Nociceptive, Radicular, and Referred Pain. BioMed Res. Int. 2018, 2018, 8793843. [CrossRef]

64. Moss, P.; Benson, H.A.E.; Will, R.; Wright, A. Patients with Knee Osteoarthritis Who Score Highly on the PainDETECT Questionnaire Present with Multimodality Hyperalgesia, Increased Pain, and Impaired Physical Function. Clin. J. Pain 2018, 34, 15-21. [CrossRef]

65. Walsh, D.A.; McWilliams, D.F.; Turley, M.J.; Dixon, M.R.; Fransès, R.E.; Mapp, P.I.; Wilson, D. Angiogenesis and nerve growth factor at the osteochondral junction in rheumatoid arthritis and osteoarthritis. Rheumatology 2010, 49, 1852-1861. [CrossRef]

66. Kaczmarek, M.; Nowakowski, A. Active Dynamic Thermography in Medical Diagnostics. In Application of Infrared to Biomedical Sciences; Springer: Singapore, 2017; pp. 291-310.

67. Vardasca, R.; Ring, F.; Plassmann, P.; Jones, C. Thermal symmetry of the upper and lower extremities in healthy subjects. Thermol. Int. 2012, 22, 53-60.

68. Ismail, E.; Merla, A. Modeling Thermal Infrared Imaging Data for Differential Diagnosis. In Application of Infrared to Biomedical Sciences; Etehadtavakol, M., Ed.; Series in BioEngineering; Springer: Singapore, 2017.

69. Saxena, A.; Ng, E.; Lim, S.T. Infrared (IR) thermography as a potential screening modality for carotid artery stenosis. Comput. Biol. Med. 2019, 113, 103419. [CrossRef]

70. Jin, C.; He, Z.-Z.; Liu, J. Finite element method based three-dimensional thermal tomography for disease diagnosis of human body. J. Heat Transfer. 2016, 138, 104501. [CrossRef]

71. Nowakowski, A. Quantitative active dynamic thermal IR-imaging and thermal tomography in medical diagnostics. In Medical Devices System, 3rd ed.; Bronzino, J.D., Ed.; Taylor \& Francis Group: Boca Raton, FL, USA, 2006; pp. 1-30.

72. Ammer, K. The Glamorgan protocol for recording and evaluation of thermal images of the human body. Thermol. Int. 2008, 18, 125-129.

73. Paszkiel, S.Z. Control, Computer Engineering and Neuroscience; Springer Nature: Chan, Germany, 2021. 
74. Skorupska, E.; Dybek, T.; Rychlik, M.; Jokiel, M.; Dobrakowski, P. The Automatization of a New Thermography Method Using Invasive Nociceptive Stimulation to Confirm an Autonomic Phenomenon within a Trigger Point Referred Pain Zone. Brain Sci. 2021, 11, 893. [CrossRef] [PubMed]

75. Maniar, N.; Bach, A.J.E.; Stewart, I.B.; Costello, J.T. The effect of using different regions of interest on local and mean skin temperature. J. Therm. Biol. 2015, 49-50, 33-38. [CrossRef] [PubMed]

76. Quesada, J.I.P.; Lucas-Cuevas, A.G.; Palmer, R.S.; Pérez-Soriano, P.; de Anda, R.M.C.O. Definition of the thermographic regions of interest in cycling by using a factor analysis. Infrared Phys. Technol. 2016, 75, 180-186. [CrossRef]

77. Fournet, D.; Ross, L.; Voelcker, T.; Redortier, B.; Havenith, G. Body mapping of thermoregulatory and perceptual responses of males and females running in the cold. J. Therm. Biol. 2013, 38, 339-344. [CrossRef]

78. Fernández-Cuevas, I.; Marins, J.C.; Carmona, P.G.; García-Concepción, M.A.; Lastras, J.A.; Quintana, M.S. Reliability and reproducibility of skin temperature of overweight subjects by an infrared thermography software designed for human beings. Thermol. Int. 2012, 22, 130-137.

79. Duarte, A.; Carrão, L.; Espanha, M.; Viana, T.; Freitas, D.; Bártolo, P.; Faria, P.; Almeida, H. Segmentation algorithms for thermal images. Procedia Technol. 2014, 16, 1560-1569. [CrossRef]

80. Barcelos, E.Z.; Caminhas, W.M.; Ribeiro, E.; Pimenta, E.M.; Palhares, R.M. A combined method for segmentation and registration for an advanced and progressive evaluation of thermal images. Sensors 2014, 14, 21950-21967. [CrossRef]

81. Ludwig, N.; Formenti, D.; Gargano, M.; Alberti, G. Skin temperature evaluation by infrared thermography: Comparison of image analysis methods. Infrared Phys. Technol. 2014, 62, 1-6. [CrossRef] 